\title{
Transvaginal sacrospinous ligament suture rectopexy for obstructed defecation symptoms: 1-year outcomes
}

\author{
Ghazaleh Rostaminia $^{1}$ (D) Steven Abramowitch ${ }^{2} \cdot$ Cecilia Chang $^{3} \cdot$ Roger P. Goldberg ${ }^{4}$
}

Received: 24 August 2020 / Accepted: 11 November 2020 / Published online: 25 November 2020

(C) The International Urogynecological Association 2020

\begin{abstract}
Introduction and hypothesis The current study was aimed at investigating the safety, efficiency, and durability of transvaginal sacrospinous ligament suture rectopexy in women with obstructed defecation symptoms (ODS) and significant rectal hypermobility/folding.

Methods This was a prospective case series study performed during December 2018 to July 2020. Women presenting to our center with pelvic organ prolapse electing for surgical treatment were screened for ODS utilizing the PFDI-20 questionnaire. Patients were eligible for inclusion if they reported OD symptoms accompanying $>50 \%$ of bowel movements (BMs), BM frequency $\geq 3$ per week, stool type 3 or 4 based on the Bristol stool chart, absence of dyssynergic Valsalva, and dynamic ultrasound indicating a rectal compression ratio $>25 \%$. Patients underwent transvaginal sacrospinous ligament rectopexy and were followed up at 2 and 12 months postoperatively.

Results A total of 20 patients underwent the procedure and completed the follow-up. Statistically significant improvements were observed in all OD symptoms and subjective improvement $(94.7 \% \pm 13.4$ and $90.6 \% \pm 18)$ at 2 and 12 months after the surgery respectively. Mean rectal compression ratio, detected via ultrasound, improved from $45.5 \% \pm 18.4$ preoperatively to $9.2 \% \pm$ 13.7 at 2 months $(p<0.0001)$ and $19.6 \% \pm 14.4$ at 12 months $(p<0.0012)$. Surgical failure, defined as combined subjective (ODS $>50 \%$ of bowel movements) and anatomical failure (rectal compression ratio $>25 \%$ ), occurred in 2 patients.

Conclusion Transvaginal sacrospinous ligament suture rectopexy was safe, feasible, and effectively treated ODS within this cohort of women undergoing POP surgery with rectal hypermobility confirmed by dynamic ultrasound.
\end{abstract}

Keywords Rectal hypermobility $\cdot$ Pelvic floor ultrasound $\cdot$ Rectal prolapse $\cdot$ Rectopexy $\cdot$ Sacrospinous ligament suspension

\section{Introduction}

Obstructed defecation symptoms (ODS) account for constipation in $7 \%$ of the adult female population [1] and are

Ghazaleh Rostaminia

ghazalerostaminia@yahoo.com

1 Female Pelvic Medicine and Reconstructive Surgery (FPMRS), Division of Urogynecology, University of Chicago Pritzker School of Medicine, NorthShore University HealthSystem, 9650 Gross Point Road, Suite 3900, Skokie, IL 60076, USA

2 Department of Bioengineering, University of Pittsburgh, Pittsburgh, PA, USA

3 NorthShore University HealthSystem Research Institute, Evanston, IL, USA

4 Division of Urogynecology, University of Chicago Pritzker School of Medicine, NorthShore University HealthSystem, Skokie, IL, USA commonly encountered in the urogynecology setting, in $\sim 60 \%$ of women presenting with pelvic organ prolapse [2]. Traditional surgical options for treating ODS have been lacking, and as a result the majority of women impacted by this condition endure their symptoms on a long-term basis. There has been a longstanding need for an effective minimally invasive surgical approach, ideally one that is mesh free and appropriate for combining with minimally invasive pelvic organ prolapse (POP) repair techniques.

Standard POP surgery techniques have unfortunately resulted in limited to modest improvements in obstructed defecation (OD) symptomology regardless of the specific surgical approach [3, 4], and specifically, the unreliable impact of posterior colporrhaphy on ODS has been established [3]. Extended CARE (E-CARE) data analyzing bowel symptoms after a 5-year follow-up concluded that abdominal sacrocolpopexy (ASC) with posterior repair appeared to have a negative impact on the recurrence of posterior wall prolapse, 
which was common at the 5-year follow-up, and resolution of ODS was no different among subjects who underwent posterior repair versus those who did not undergo posterior repair at the time of ASC [5].

With the advent of modern pelvic floor imaging modalities, the association between rectal support defects and the presence of ODS has been established [6]. These defects in rectal support are independent of posterior vaginal wall prolapse representing a "sliding" or "folding" defect of the rectum separate and unrelated to the vaginal plane, and may be referred to as rectal intussusception, rectal prolapse or simply "rectal hypermobility." The term "rectal hypermobility" is applied to describe this condition based on the appearance of rectal tube movement visualized on dynamic imaging techniques such as MR defecography and dynamic posterior compartment ultrasound. These rectal support defects are clinically unrelated to the presence or absence of a rectocele [6] — consistent with unsatisfactory cure rates for ODS among patients undergoing traditional POP surgery. Despite the known lack of association between posterior vaginal wall support and ODS, there nonetheless remains a persistent trend among pelvic surgeons to manage ODS by repairing the posterior vaginal compartment — perhaps in part because rectoceles "seem" like a logical reason for stool entrapment, and perhaps also because more attractive and effective solutions do not yet exist. For many patients this approach may address the wrong surgical plane, disregard the underlying rectal support defect accounting for the defecatory symptoms, and lead to a disappointing clinical outcome.

Ventral mesh rectopexy (VR), in contrast, has gained increasing acceptance for the surgical correction of rectal prolapse and high-grade internal rectal intussusception. Anterior mobilization of the distal rectum and mesh suspension performed during VR can correct full-thickness rectal prolapse, rectoceles, and internal rectal prolapse. Rectopexy is traditionally performed via an abdominal approach with favorable long-term results for the treatment of rectal prolapse with low recurrence rates and no change in functional outcomes regarding fecal incontinence [5]. Several reports have concluded that ventral rectopexy can be successfully performed at the time of sacrocolpopexy for patients with POP accompanied by ODS and rectal prolapse or intussusception $[7,8]$. This technique addresses the descent of the posterior and middle compartments combined with reinforcement of the vaginal septum and elevation of the pelvic floor $[1,9,10]$. However, because most existing rectopexy techniques utilize mesh and require an abdominal approach, this operation tends to be applicable for a relatively small percentage of patients who are impacted by OD and are comfortable with its associated risks and benefits. Likewise, from the surgeon's perspective this surgical approach has been limited to mostly colorectal specialists and has not gained popularity as a routine offering among the majority of reconstructive pelvic surgeons.
Transvaginal sacrospinous ligament fixation (SSLF) for vaginal vault prolapse has been shown to offer good longterm anatomical results with excellent maintenance of vaginal vault suspension and a high degree of patient satisfaction [4]. Our center has developed surgical techniques centering on SSLF for more than two decades $[11,12]$, and as a natural progression of this work we considered whether the SSLF technique may provide an opportunity to address rectal support defects underlying ODS. Our interest increased as we utilized dynamic pelvic ultrasound studies that demonstrated discrete rectal support defects to be an extremely reliable predictor of ODS in women [6]. Moreover, these ultrasound works also suggested that the pivotal site of rectal hypermobility in patients with ODS might be located in an extremely predictable anatomical location, at the level of the sacrospinous ligaments (SSLs; Fig. 1). On the basis of several hundred ultrasound studies performed on patients with and without ODS, combined with cadaver dissection and consideration of our existing minimally invasive SSLF techniques, we concluded that the SSL may represent an anatomically and physiologically appropriate anchoring site for a novel "transvaginal rectopexy" approach for ODS. By addressing the core anatomical deficiency underlying ODS utilizing an efficient, mesh-free transvaginal approach, we hoped to identify a solution with a more favorable risk-benefit balance than traditional rectopexy methods, one that could be more widely utilized among the large population of women silently suffering with ODS.

This study was aimed at investigating the safety, feasibility, and durability of transvaginal SSL suture rectopexy in women with ODS who had significant rectal hypermobility based on pelvic floor dynamic imaging defined as a compression ratio $>$ $25 \%$. In the present analysis we report on 2- and 12-month subjective and objective outcomes of this novel procedure for the treatment of ODS.

\section{Materials and methods}

This prospective surgical feasibility case series study was performed during the period December 2018 to July 2020, and was approved by our institutional review board. Women presenting to our tertiary urogynecology center with pelvic organ prolapse symptoms, and who were also scheduled for surgical treatment, were screened for ODS utilizing their responses to the PFDI-20 [13] completed during their initial office visit. Patients answering "yes" to questions $4,7,8$, or 14 on the PFDI-20 were offered a referral to our Women's Bowel Clinic for further assessment. These questions are "Ever have to push on the vagina or around the rectum to have or complete a bowel movement?", "Feel you need to strain too hard to have a bowel movement?," "Feel you have not completely emptied your bowels at the end of a bowel movement?", and 
Fig. 1 a 3D model created based on MR imaging of a normal healthy volunteer. The anatomical location of the rectum relative to the sacrospinous ligament and the suturing points are demonstrated. b A sagittal view demonstrating the anatomical landmark on the rectum at the level of the sacrospinous ligament
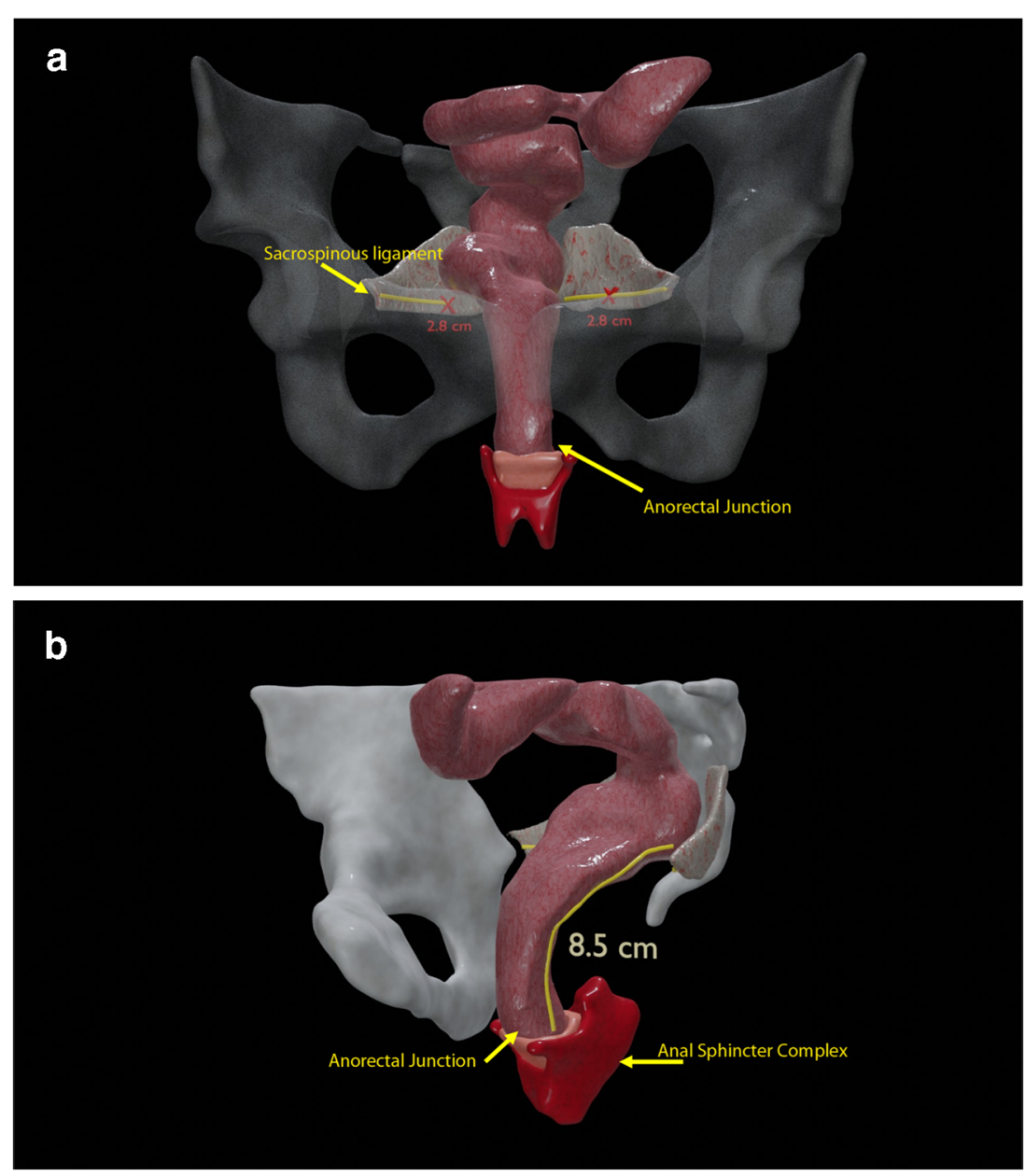

"Does part of your bowel ever pass through the rectum and bulge outside during or after a bowel movement?"

During the bowel clinic visit, all patients completed an interview with detailed review of bowel symptoms and habits, pelvic examination, and pelvic floor $3 \mathrm{D}$ and dynamic ultrasound. Inclusion criteria included the following: (1) OD symptoms accompanying more than $50 \%$ of bowel movements (BM), (2) reported BM frequency $\geq 3$ per week, (3) stool type 3 or 4 based on the Bristol stool chart, (4) absence of dyssynergic Valsalva, uncoordinated contraction of pelvic floor muscles during Valsalva, and (5) ultrasound indicating rectal compression ratio of more than $25 \%$. Our exclusion criteria were history of colorectal malignancy, or colorectal surgeries. Women meeting these inclusion criteria were invited to participate in the study and after informed consent, were enrolled in the study. Compression ratio was calculated by the percentage change in rectovaginal septum length from rest to maximum Valsalva [6]; this quantitative measure of rectal hypermobility / intussusception has been previously validated and published by our center [6, 14] and is routinely utilized within our treatment algorithm to diagnose ODS with underlying rectal hypermobility/folding. Prior work has outlined the correlation between abnormal rectal compression ratio and
OD symptoms, with a threshold of $\geq 25 \%$ indicating 4 times greater odds of having severe symptoms [14].

Subjects underwent follow-up at 2 and 12 months postoperatively for the assessment of symptoms, physical examination, and pelvic floor ultrasound with calculation of rectal compression ratio to evaluate the impact of surgery on rectal hypermobility.

\section{Dynamic posterior compartment ultrasound technique}

The pelvic floor ultrasound techniques have been described previously [15].

Imaging was obtained using the BK5000 (BK Medical, Peabody, MA, USA) X14L4 12-MHz transducer. All ultrasound studies were performed in the office setting with the patient in the dorsal lithotomy position, with hips flexed and abducted. No preparation was required and no rectal or vaginal contrast agent was used. Patients were instructed to arrive at the office with a partially full bladder and, to avoid excessive pressure on surrounding structures that might distort the anatomy, the probe was inserted into the vagina in a neutral 
position. The dynamic ultrasound videos taken during Valsalva were saved for further analysis.

The distance between the posterior cul de sac and anorectal junction ("rectovaginal septum length") was measured both at rest and during Valsalva straining efforts using a dynamic imaging protocol in the midsagittal plane, allowing posterior compartment structures to be visualized (Fig. 2). It should be noted that for Valsalva straining efforts, patients were instructed to relax their pelvic floor while increasing intra-abdominal pressure. All images included the cul de sac apically and the levator plate/anorectal junction caudally in order to standardize framing of the anatomy. The dynamic recording was started with the patient at rest and captured for $5 \mathrm{~s}$ during Valsalva straining.

"Compression ratio" was calculated as a means of quantifying the relative change in length of the rectovaginal septum (RVS); in other words, the degree of hypermobility - also referred to as a "sliding rectum"- -was expressed as a percentage using the following formula: compression ratio $=100 *$
(RVS length at rest - RVS septum length at Valsalva) / RVS length at rest [6].

\section{Surgical technique}

The posterior vaginal wall dissection started with a horizontal incision in the mid-posterior vaginal wall. Dissection was carried down to the SSL bluntly on each side. SSL sutures were placed using a push-and-catch suturing device (Capio Slim; Boston Scientific Corporation, USA) to deliver a single 0polydiaxone suture (Monodek, Teleflex Medical OEM) into each ligament approximately $2-2.5 \mathrm{~cm}$ medial to the ipsilateral ischial spine. With one finger inside the rectum, the suture was passed through the lateral rectal ligament and rectal muscularis layer, as a two-bite running suture, at a point 7 $8 \mathrm{~cm}$ cephalad to the anal verge. After tying down the knot on the first side, rectal suture placement was then performed on the contralateral side; this sequential suturing and tying down of left- and right-sided rectal suspensions allowed for the

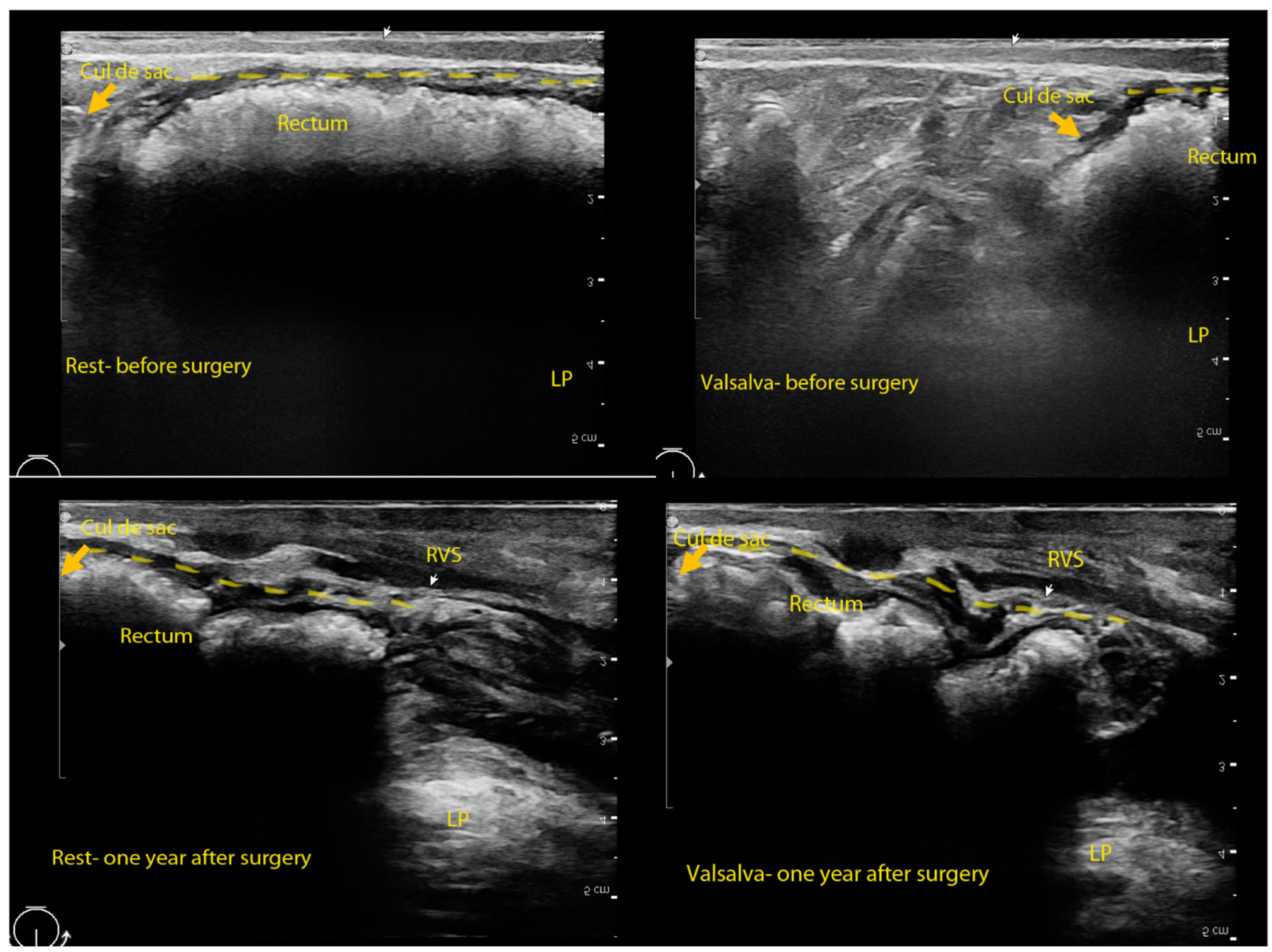

Fig. 2 a Posterior compartment dynamic ultrasound of a patient at rest and maximum Valsalva before surgery. b Ultrasound imaging of the same patient at rest and maximum Valsalva after rectopexy and at the 12-month follow-up visit. $L P$ levator plate, $R V S$ rectovaginal septum 
assessment and fine-tuning of the rectal suspension and avoidance of excess tension or stretching of the rectal wall.

After suspension of the rectum bilaterally, three additional interrupted 0-polydiaxone sutures were used to reattach the midline posterior vaginal wall to the midline anterior rectal wall at the same level (7-8 cm cephalad from the anal verge); these sutures provided reinforcement and tension relief to the midline rectum between each lateral SSL suspension suture, and also served to close a potential enterocele space. The anatomical landmarks used for this procedure are demonstrated in Fig. 1.

\section{Definition of surgical failure}

"Subjective failure" was defined as the presence of OD symptoms in more than $50 \%$ of bowel movements as reported by the patient and "anatomical failure" was defined as the presence of rectal hypermobility based on a compression ratio $>25 \%$ on dynamic ultrasound. "Surgical failure" was defined as a composite outcome that included both subjective and anatomical failure.

\section{Statistical methods}

Our power calculation indicated that a total of 20 patients were required to achieve $80 \%$ power to determine $50 \%$ improvement of symptoms from baseline to 2- and 12-month postoperative follow-up using an effect size of 0.7 and two-tailed alpha of 0.05 .

Pelvic organ prolapse quantification (POP-Q) examination values and dynamic ultrasound findings were compared at enrollment, and at the 2- and 12-month follow-up. Subjects were categorized as either surgical success or failure at both 2 and 12 months. Continuous data were compared using paired and unpaired $t$ tests, and Chi-squared or Fisher's exact test was used to evaluate categorical data, with $p<0.05$ considered statistically significant. All statistical analyses were performed using SAS 9.4 (SAS Institute, Cary, NC, USA).

\section{Results}

Twenty subjects underwent transvaginal SSL rectopexy and have been evaluated at both 2- and 12-month follow-up visits. One subject at 2 months and 4 subjects at 12 months were lost to follow-up. Two others were unable to follow up in person owing to the COVID-19 pandemic but completed a telephone interview.

All subjects were white and the mean age within the cohort was $61.1 \pm 11.5$ years old. Five patients had undergone previous abdominal hysterectomy and 5 had a history of prior pelvic floor reconstructive surgery. During the surgical intervention, 3 patients underwent concomitant bilateral SSL vaginal cuff suspension, 5 underwent vaginal hysterectomy and uterosacral ligament suspension, 13 underwent anterior repair, and 9 underwent posterior repair. Fourteen patients received mid-urethral slings.

\section{Intra- and postoperative complications}

One patient (5\%) required removal of one SSL suture, and one $(5 \%)$ required removal of both (bilateral) SSL sutures on postoperative days 4 and 2 respectively, owing to severe gluteal pain. No other complications were encountered during and/or after the procedure. There were no readmissions.

\section{Surgical outcome}

The prevalence of ODS specific symptoms, POP-Q examination and pelvic floor dynamic ultrasound findings at enrollment, and at 2 and 12 months after surgery, are summarized in Table 1.

Statistically significant improvements were seen for all OD symptoms and subjective improvement outcomes $(94.7 \% \pm$ 13.4 and $90.6 \% \pm 18)$ at 2 and 12 months after surgery. Ultrasound evaluation at postoperative follow-up indicated that the rectal compression ratio improved significantly alongside the symptoms, in comparison with the preoperative baseline, with an improvement from $45.5 \% \pm 18.4$ preoperatively to $9.2 \% \pm 13.7$ ( 2 months, $p<0.0001$ ) and $19.6 \% \pm 14.4$ (12 months, $p<0.0012$ ) postoperatively. Although there was an increase in rectal hypermobility observed from 2 months to 12 months, based on mean compression ratios calculated during those two follow-up points, the value did not reach the threshold of $25 \%$ that we have previously defined as clinically significant and was not statistically significant either.

All but one subject (19 out of 20,95\%) reported subjective success (absence of OD symptoms in more than $50 \%$ of bowel movements) and all subjects were found to have anatomical success (absence of rectal compression ratio $>25 \%$ ) at 2 months. At 12 months postoperatively, 2 patients (10\%) reported OD symptoms in more than 50\% of the BMs (including the same symptomatic patient identified at the 2-month follow-up visit) and dynamic ultrasound revealed a compression ratio above $25 \%$ in both cases.

Two other asymptomatic subjects (10\%) at the 12-month follow-up were found to have a $30 \%$ compression ratio; in both cases this represented a significant improvement from their baseline compression ratio ( $80 \%$ for both subjects), and they remained asymptomatic and free of OD symptoms. Notably, one of these subjects had complete external rectal prolapse (protrusion $6 \mathrm{~cm}$ outside anus), which was also diagnosed after induction of anesthesia in the operating room; the rectal prolapse resolved following transvaginal rectopexy and this patient has remained completely asymptomatic up to 1 year postoperatively (Fig. 2). 
Table 1 Summary of preoperative and postoperative symptoms, examination, and ultrasound measurements. Comparison between the groups of patients with surgical success and failure at 2 and 12 months

\begin{tabular}{|c|c|c|c|c|c|c|c|c|}
\hline & \multirow{2}{*}{$\begin{array}{l}\text { Preoperative } \\
(n=20)\end{array}$} & \multicolumn{2}{|l|}{2 months $(n=19)$} & \multicolumn{3}{|c|}{12 months $(n=16)$} & \multirow[t]{2}{*}{$p$ value $^{\mathrm{b}}$} & \multirow[t]{2}{*}{$n$ value $^{\mathrm{c}}$} \\
\hline & & $\begin{array}{l}\text { Surgical success } \\
(n=19)\end{array}$ & $\begin{array}{l}\text { Surgical failure } \\
(n=0)\end{array}$ & $\begin{array}{l}\text { Surgical success } \\
(n=14)\end{array}$ & $\begin{array}{l}\text { Surgical failure } \\
(n=2)\end{array}$ & $p$ value $\mathrm{a}^{\mathrm{a}}$ & & \\
\hline $\begin{array}{l}\text { Subjective improvement } \\
\quad(\%) \text {, mean } \pm \text { SD }\end{array}$ & & $94.74 \pm 13.38$ & & & & & & \\
\hline Age (years), mean $\pm \mathrm{SD}$ & $61.10 \pm 11.49$ & & & & & & & \\
\hline $\begin{array}{l}\mathrm{BM} \text { frequency/week, } \\
\text { mean } \pm \mathrm{SD}\end{array}$ & $7.47 \pm 7.65$ & $9.58 \pm 5.03$ & & $7.57 \pm 3.55$ & $7.50 \pm 0.71$ & 0.9784 & 0.2803 & 0.3420 \\
\hline \multicolumn{9}{|l|}{ Stool type, $n(\%)$} \\
\hline $\begin{array}{l}\text { Too hard }(1,2) \\
\text { Normal }(3,4,5)\end{array}$ & $\begin{array}{l}3(16.67) \\
14(77.78)\end{array}$ & $\begin{array}{l}1(5.26) \\
17(89.47)\end{array}$ & & $\begin{array}{l}0(0.00) \\
14(100.00)\end{array}$ & $\begin{array}{l}0(0.00) \\
2(100.00)\end{array}$ & - & 0.5724 & - \\
\hline Loose $(6,7)$ & $1(5.56)$ & $1(5.26)$ & & $0(0.00)$ & $0(0.00)$ & & & \\
\hline \multicolumn{9}{|l|}{ Straining, $n(\%)$} \\
\hline $\begin{array}{l}\text { Yes } \\
\text { No }\end{array}$ & $\begin{array}{l}18(90.00) \\
2(10.00)\end{array}$ & $\begin{array}{l}1(5.26) \\
18(94.74)\end{array}$ & & $\begin{array}{l}0(0.00) \\
14(100.00)\end{array}$ & $\begin{array}{l}0(0.00) \\
2(100.00)\end{array}$ & - & $<0.0001$ & - \\
\hline $\begin{array}{l}\text { Incomplete emptying, } \\
\text { mean } \pm \text { SD }\end{array}$ & $62.83 \pm 25.63$ & $4.47 \pm 12.57$ & & $3.57 \pm 8.42$ & $45.00 \pm 7.07$ & $<0.0001$ & $<0.0001$ & $<0.0001$ \\
\hline $\begin{array}{l}<50 \% \\
\geq 50 \%\end{array}$ & $\begin{array}{l}2(11.11) \\
16(88.89)\end{array}$ & $\begin{array}{l}18(94.74) \\
1(5.26)\end{array}$ & & $\begin{array}{l}14(100.00) \\
0(0.00)\end{array}$ & $\begin{array}{l}1(50.00) \\
1(50.00)\end{array}$ & 0.1250 & 0.0002 & 0.0009 \\
\hline \multicolumn{9}{|c|}{ Splinting inside rectum, $n(\%)$} \\
\hline $\begin{array}{l}\text { Yes } \\
\text { No }\end{array}$ & $\begin{array}{l}4(20.00) \\
16(80.00)\end{array}$ & $\begin{array}{l}1(5.26) \\
18(94.74)\end{array}$ & & $\begin{array}{l}1(7.14) \\
13(92.86)\end{array}$ & $\begin{array}{l}0(0.00) \\
2(100.00)\end{array}$ & 1.0000 & 0.1797 & 0.3173 \\
\hline \multicolumn{9}{|c|}{ Splinting inside vagina, $n(\%)$} \\
\hline $\begin{array}{l}\text { Yes } \\
\text { No }\end{array}$ & $\begin{array}{l}4(20.00) \\
16(80.00)\end{array}$ & $\begin{array}{l}0(0.00) \\
19(100.00)\end{array}$ & & $\begin{array}{l}0(0.00) \\
14(100.00)\end{array}$ & $\begin{array}{l}1(50.00) \\
1(50.00)\end{array}$ & 0.1250 & - & 0.0833 \\
\hline \multicolumn{9}{|c|}{ Splinting around rectum, $n(\%)$} \\
\hline $\begin{array}{l}\text { Yes } \\
\text { No }\end{array}$ & $\begin{array}{l}10(50.00) \\
10(50.00)\end{array}$ & $\begin{array}{l}0(0.00) \\
19(100.00)\end{array}$ & & $\begin{array}{l}0(0.00) \\
14(100.00)\end{array}$ & $\begin{array}{l}0(0.00) \\
2(100.00)\end{array}$ & - & - & - \\
\hline \multicolumn{9}{|c|}{ Splinting on perineum, $n(\%)$} \\
\hline $\begin{array}{l}\text { Yes } \\
\text { No }\end{array}$ & $\begin{array}{l}4(20.00) \\
16(80.00)\end{array}$ & $\begin{array}{l}0(0.00) \\
19(100.00)\end{array}$ & & $\begin{array}{l}1(7.14) \\
13(92.86)\end{array}$ & $\begin{array}{l}0(0.00) \\
2(100.00)\end{array}$ & 1.0000 & - & 0.5637 \\
\hline $\mathrm{Ba}$, mean $\pm \mathrm{SD}$ & $-0.54 \pm 1.11$ & $-1.82 \pm 0.51$ & & $-1.79 \pm 0.66$ & $-1.50 \pm 0.71$ & 0.5736 & 0.0003 & 0.0022 \\
\hline $\mathrm{C}$, mean $\pm \mathrm{SD}$ & $-3.90 \pm 3.57$ & $-6.84 \pm 0.50$ & & $-6.33 \pm 0.89$ & $-7.00 \pm 0.00$ & 0.0246 & 0.0017 & 0.0128 \\
\hline $\mathrm{GH}$, mean $\pm \mathrm{SD}$ & $3.88 \pm 0.78$ & $3.32 \pm 0.42$ & & $3.33 \pm 0.44$ & 5.00 & - & 0.0107 & 0.0269 \\
\hline $\mathrm{PB}$, mean $\pm \mathrm{SD}$ & $2.70 \pm 0.44$ & $3.29 \pm 0.54$ & & $3.67 \pm 0.33$ & $3.25 \pm 0.35$ & 0.1222 & 0.0006 & $<0.0001$ \\
\hline $\mathrm{Bp}$, mean $\pm \mathrm{SD}$ & $-1.00 \pm 1.19$ & $-2.24 \pm 0.45$ & & $-2.21 \pm 0.33$ & $-0.75 \pm 1.77$ & 0.4503 & 0.0002 & 0.0018 \\
\hline $\begin{array}{l}\text { RVS length at rest, } \\
\text { mean } \pm \mathrm{SD}\end{array}$ & $4.66 \pm 1.12$ & $5.13 \pm 0.78$ & & $4.63 \pm 0.77$ & $5.00 \pm 0.00$ & 0.1207 & 0.0082 & 0.8853 \\
\hline $\begin{array}{l}\text { RVS length at strain, } \\
\text { mean } \pm \text { SD }\end{array}$ & $2.66 \pm 1.09$ & $4.68 \pm 1.13$ & & $4.00 \pm 0.85$ & $2.75 \pm 0.35$ & 0.0700 & $<0.0001$ & 0.0055 \\
\hline $\begin{array}{l}\text { Compression ratio }(\%) \\
\text { mean } \pm \mathrm{SD}\end{array}$ & $45.53 \pm 18.40$ & $9.21 \pm 13.67$ & & $15.33 \pm 10.16$ & $45.00 \pm 7.07$ & 0.0021 & $<0.0001$ & 0.0012 \\
\hline
\end{tabular}

$B M$ bowel movements, $G H$ genital hiatus, $P B$ perineal body, $R V S$ rectovaginal septum

${ }^{\text {a }}$ Comparison between surgical success and surgical failure at 12 months

${ }^{\mathrm{b}}$ Comparison between preoperative and 2 months

${ }^{\mathrm{c}}$ Comparison between preoperative and 12 months

$P$ value $<0.05$ is marked in bold

Utilizing a composite definition for surgical success that included both subjective (ODS in $<50 \%$ of BMs) and anatomical (compression ratio $<25 \%$ ) outcomes, 2 patients were defined as surgical failure (Table 1). No statistical differences were observed in baseline demographics, physical examination, preoperative ultrasound findings, and performance of 
posterior colporrhaphy between the surgical failure group and the surgical success group.

\section{Discussion}

Evaluation of this surgical cohort at 1 year suggests that transvaginal SSL suture rectopexy represents a safe, feasible, and highly effective procedure for the treatment of OD symptoms associated with rectal laxity. Surgical success appeared to be independent and unrelated to the presence or absence of a posterior vaginal POP bulge, and likewise, concomitant vaginal prolapse surgeries including rectocele/posterior compartment repairs had no apparent impact on rectopexy outcome. Although the current study was focused on the management of occult rectal prolapse/intussusception, we encountered one case of overt rectal prolapse; this patient had an excellent outcome with complete resolution of her anal bulge during defecation at both 2 and 12 months postoperatively (Fig. 2). Although anecdotal, this outlier within our cohort raises our level of interest in potentially exploring the application of this new surgical method to treat patients with more advanced/ externalized rectal prolapse.

The procedure has thus far proven to be safe and very efficient, and we have observed no major complications such as infection, hemorrhage, rectal injury, or readmission. Minor complications within this cohort were limited to the removal of SSL sutures in 2 patients owing to severe gluteal pain. Postoperative gluteal pain is a known complication for any SSLF suspension, and the nature of pain and resolution after suture removal in these two rectopexy patients followed a typical course that we occasionally observe after suturebased SSLF performed for vaginal prolapse. These two cases occurred early in our experience, and in the interim we have refined our technique to emphasize suture placement into the medial aspect of the SSL, and also to avoid excess pressure between the suturing device and the ligament surface. We continue to perform sacrospinous rectopexy at our center, and have not had any additional cases requiring suture removal to date.

Over the past century, more than 100 different surgical techniques have been described to repair rectal prolapse [16, 17]. These procedures can be categorized into abdominal and perineal procedures [18]. In general, perineal surgical repairs cause less morbidity and mortality, but are attributed with greater recurrences when compared with abdominal operations $[19,20]$. Our suture-based transvaginal rectopexy method is minimally invasive, requiring no abdominal incision, no entry into the peritoneal cavity, no mesh or graft implant, and no disruption of the rectal lumen. We believe that these characteristics make it a potentially safer and more widely applicable technique compared with traditional abdominal or transrectal approaches; ideally, comparison of this novel transvaginal method with traditional techniques would be analyzed in a prospective randomized trial.

The most similar previous trial to ours was published by Gurland et al. [21] who evaluated the feasibility of fixation of the rectum to the SSL via a transvaginal approach, utilizing a Surgisis biological graft (Cook Medical, Bloomington, IN, USA) because of the expectation of poor tissue structure in their patient population and in order to avoid the risks that may be associated with a non-absorbable mesh. In that trial, the graft was attached to the anterior rectal wall and also to the SSL on each side. Seven patients underwent the procedure and the authors concluded that transvaginal sacrospinous rectopexy was a safe, minimally invasive, technically feasible technique for the treatment of rectal prolapse. In contrast to this trial, our method utilizes native tissue without an additional graft or mesh, and our patient selection and identification of rectal hypermobility is guided by the use of dynamic posterior compartment ultrasound.

We strongly believe that patients with combined vaginal and rectal prolapse seeking care should be offered multidisciplinary pelvic floor evaluation and, whenever feasible, the opportunity for an efficient combined surgery to address both POP and colorectal symptoms. Similar pelvic dissections are required for transvaginal rectopexy and vaginal apex SSL suspension; therefore, in our experience, combining transvaginal POP repair with rectopexy adds little operative time and results in a more complete pelvic floor repair. Arguably, the standard of care for surgeons managing women with pelvic floor disorders should include recognition of overlapping POP and colorectal symptoms, accurate diagnosis of the underlying anatomical issues, and development of a surgical plan that provides maximum symptom relief in an efficient and safe manner. We believe we have identified a simple and effective diagnostic and surgical approach which may allow most pelvic reconstructive surgeons to better identify and manage ODS in women. Utilizing a quick and painless dynamic ultrasound study performed in the office, combined with a minimally invasive surgical solution that can be conveniently combined with vaginal POP surgery, we now manage ODS successfully while avoiding traditional imaging modalities and more invasive surgical treatment options that we previously relied upon.

This feasibility study involved a relatively small population and was conducted by only one center with longstanding experience in SSLF procedures. Determining outcomes in a larger group, and/or with a more heterogeneous group of surgeons, will require further study. We also lost $20 \%$ of patients to follow-up, in large part because of the pandemic situation resulting in patients being hesitant to present in the office for follow-up visits. The increasing compression ratio trend observed from 2 to 12 months should also be further investigated to determine if rectal support remains stable, or continues to weaken and lead to recurrence of OD symptoms, over longer 
time periods. Based on the success of this feasibility study, we hope to conduct future trials comparing transvaginal rectopexy with other procedures available for the treatment of ODS and rectal prolapse.

Rectal compression ratio, a novel measure of occult rectal hypermobility developed at our center that can be obtained within seconds using dynamic ultrasound, proved to correlate with surgical success. Significant ODS symptoms correlated closely with abnormal compression ratios before surgery, and conversely, subjectively satisfied patients after surgery demonstrated normalized compression ratios. In other words, objective repair and stabilization of the underlying rectal support defect correlated impressively with patient satisfaction and symptom relief.

In conclusion, this prospective surgical trial evaluated the safety and effectiveness of a minimally invasive, mesh-free transvaginal rectopexy. Our initial experience up to 1 year indicated that the operation was safe and resulted in a consistent outcome when performed by our team, who have a significant amount of prior experience with SSLF procedures for POP. The surgery successfully suspended the rectum, as demonstrated by the resolution of OD symptoms in nearly all subjects, and the correction of rectal hypermobility according to pre- and postoperative dynamic ultrasound assessment. In our view, this technique has the potential to replace more invasive rectopexy approaches, such as perineal and meshaugmented abdominal techniques. If ongoing study further establishes this method as a quick, safe, mesh-free transvaginal option providing consistent relief of OD, then we believe a large population of women may benefit from a new option for this bothersome and often ignored pelvic floor symptom.

\section{Compliance with ethical standards}

\section{Conflicts of interest None.}

\section{References}

1. D'Hoore A, Penninckx F. Obstructed defecation. Colorectal Dis. 2003;5(4):280-7.

2. Rostaminia G, Abramowitch S, Chang C, Goldberg RP. The role of conventional pelvic floor reconstructive surgeries in obstructed defecation symptoms change: CARE and OPTIMAL trials subanalysis of 2-year follow-up data. Int Urogynecol J. 2019;31(7): 1325-1334.

3. Kahn MA, Stanton SL. Posterior colporrhaphy: its effects on bowel and sexual function. BJOG. 1997;104(1):82-6.

4. Karlbom U, Graf W, Nilsson S, Påhlman L. Does surgical repair of a rectocele improve rectal emptying? Dis Colon Rectum. 1996;39(11):1296-302.
5. Grimes C, Lukacz ES, Gantz MG, Warren LK, Brubaker L, Zyczynski HM, et al. What happens to the posterior compartment and bowel symptoms after sacrocolpopexy? Evaluation of 5-year outcomes from E-CARE. Female Pelvic Med Reconstruct Surg. 2014;20(5):261.

6. Rostaminia G, Abramowitch S, Chang C, Goldberg RP. Descent and hypermobility of the rectum in women with obstructed defecation symptoms. Int Urogynecol J. 2019;31(2):337-349.

7. Lamb SV, Massengill J, Sheridan MJ, Stern LE, von Pechmann W. Safety of combined abdominal sacral colpopexy and sigmoid resection with suture rectopexy: a retrospective cohort study. Female Pelvic Med Reconstruct Surg. 2015;21(1):18-24.

8. Slawik S, Soulsby R, Carter H, Payne H, Dixon A. Laparoscopic ventral rectopexy, posterior colporrhaphy and vaginal sacrocolpopexy for the treatment of recto-genital prolapse and mechanical outlet obstruction. Colorectal Dis. 2008;10(2):138-43.

9. Gurland B. Ventral mesh rectopexy: is this the new standard for surgical treatment of pelvic organ prolapse? Dis Colon Rectum. 2014;57(12):1446-7.

10. D'Hoore A, Cadoni R, Penninckx F. Long-term outcome of laparoscopic ventral rectopexy for total rectal prolapse. Br J Surg. 2004;91(11):1500-5.

11. Goldberg RP, Tomezsko JE, Winkler HA, Koduri S, Culligan PJ, Sand PK. Anterior or posterior sacrospinous vaginal vault suspension: long-term anatomic and functional evaluation. Obstet Gynecol. 2001;98(2):199-204.

12. Botros SM, Goldberg RP, Sand PK. Sacrospinous ligament suspension for vaginal vault prolapse. In: Raz S, Rodriguez LV, editors. Female urology. Philadelphia: Saunders. 2008. p. 673-82.

13. Barber MD. Questionnaires for women with pelvic floor disorders. Int Urogynecol J. 2007;18(4):461-5.

14. Rostaminia G, Abramowitch S, Goldberg RP. Obstructed defecation symptom severity and degree of rectal hypermobility detected by dynamic ultrasound. Abstract, AUGS/IUGA, Nashville, USA. 2019.

15. Rostaminia G, White DE, Quiroz LH, Shobeiri SA. Levator plate descent correlates with levator ani muscle deficiency. Neurourol Urodyn. 2015;34(1):55-9.

16. Formijne Jonkers H, Draaisma W, Wexner S, Broeders I, Bemelman W, Lindsey I, et al. Evaluation and surgical treatment of rectal prolapse: an international survey. Colorectal Dis. 2013;15(1):115-9.

17. Laubert T, Kleemann M, Schorcht A, Czymek R, Jungbluth T, Bader FG, et al. Laparoscopic resection rectopexy for rectal prolapse: a single-center study during 16 years. Surg Endosc. 2010;24(10):2401-6.

18. Ehlig BE, Sullivan ES. The modified Delorme operation: its place in surgical treatment for massive rectal prolapse. Dis Colon Rectum. 1979;22(8):513-21.

19. Makineni H, Thejeswi P, Rai BS. Evaluation of clinical outcomes after abdominal rectopexy and Delorme's procedure for rectal prolapse: a prospective study. J Clin Diagn Res. 2014;8(5):NC04.

20. Penninckx F, D'Hoore A, Sohier S, Kerremans R. Abdominal resection rectopexy versus Delorme's procedure for rectal prolapse: a predictable outcome. Int J Colorectal Dis. 1997;12(1):49-50.

21. Gurland B, Garrett K, Firoozi F, Goldman H. Transvaginal sacrospinous rectopexy: initial clinical experience. Tech Coloproctol. 2010;14(2):169-73.

Publisher's note Springer Nature remains neutral with regard to jurisdictional claims in published maps and institutional affiliations. 